\title{
Why does the spotted UX Ari system get bluer as it gets darker?
}

\author{
V. Aarum Ulvås and O. Engvold \\ Institute of Theoretical Astrophysics, PO Box 1029 Blindern, 0315 Oslo, Norway \\ Received 2 October 2002 / Accepted 12 January 2003

\begin{abstract}
We present simplified calculations of UX Ari $B-V$ colour index and $V$ magnitude for a range of parameter values. The results of our calculations are compared to previously published measurements. We find that our calculations reproduce the well established colour-brightness relation (UX Ari gets bluer as it gets darker) only if the dark spots on the surface of the $\mathrm{K}$ star are accompanied by bright facular areas. There is currently an ambiguity between the temperature of the facular areas and the portion of the stellar surface occupied by them.
\end{abstract}

Key words. stars: activity - binaries: spectroscopic - stars: individual: UX Ari - stars: late-type - starspots

\section{Introduction}

UX Ari (HD 21242) is a spectroscopic triple system (Duemmler \& Aarum 2001, and references therein) consisting of a RS CVn binary and a faint third star. The primary and secondary components of the RS CVn-type binary have spectral class K0 IV and G5 V, respectively (Carlos \& Popper 1971). It is listed in the catalogue of chromospherically active binary stars (CABS 28; Strassmeier et al. 1993) and the Hipparcos catalogue (HIP 16042; ESA 1997).

Previously published UX Ari photometry has revealed a distinct relation between the $B-V$ colour index and the $V$ magnitude; the UX Ari system becomes bluer as it becomes fainter (Zeilik et al. 1982; Wacker et al. 1986; Mohin \& Raveendran 1989; Strassmeier et al. 1989; Rodonò \& Cutispoto 1992; Raveendran \& Mohin 1995; Aarum Ulvås \& Henry 2003). As an illustration of this puzzling relation, we have plotted the $B-V$ colour index and the $V$ magnitude against orbital phase for a typical observing season in Fig. 1. The cause of this colour-brightness relation is currently under debate. Two explanations that have been brought forward, are (1) the bluer flux of the $\mathrm{G}$ star becoming more dominant as the $\mathrm{K}$ star becomes more spotted (Wacker et al. 1986; Mohin \& Raveendran 1989; Raveendran \& Mohin 1995), and (2) dark spots on the surface of the K star being accompanied by bright facular or plage-like areas (Rodonò \& Cutispoto 1992).

In this letter, we present theoretical calculations of $B-$ $V$ and $V$ relative to the unspotted case for varying spot area, spot temperature, and area and temperature of faculae. The results of the calculations are compared to previously published measurements (Hall et al. 1975; Hall 1977; Sarma \& Prakasa Rao 1984; Mohin \& Raveendran 1989;

Send offprint requests to: V. Aarum Ulvås, e-mail: v.a.ulvas@astro.uio.no
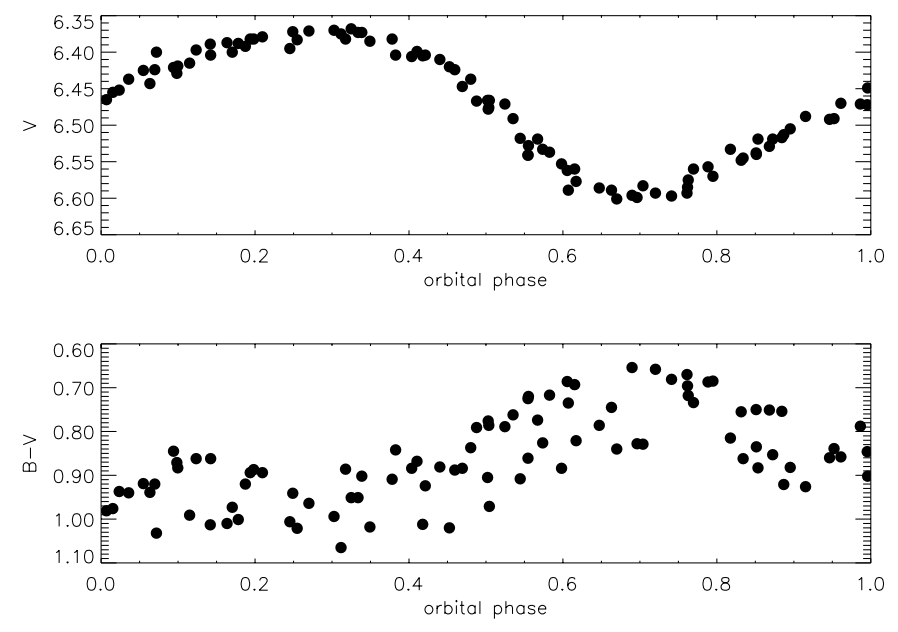

Fig. 1. The UX Ari colour-brightness relation. The relatively larger scatter in $B-V$ comes from stronger noise in the $B$ measurements. The orbital phases were calculated from the ephemeris of Duemmler \& Aarum (2001).

Strassmeier et al. 1989; Raveendran \& Mohin 1995; Padmakar \& Pandey 1996; Aarum Ulvås \& Henry 2003).

\section{Simplified photometric models of UX Ari}

We have calculated the difference in $V$ and the difference in $B-V$ for the whole UX Ari system compared to the case of unspotted $\mathrm{K}$ star. The calculations resulted in $\Delta V$ and $\Delta(B-V)$ in the sense spotted minus unspotted. In the following, we describe briefly the two methods we have used and present our results. 
Table 1. Parameters of the UX Ari system used in our model calculations. The facular temperature $T_{\mathrm{f}}$ was tentatively chosen to be $250 \mathrm{~K}$ higher than the $\mathrm{K}$ star effective temperature $T_{\mathrm{K}}$. The quoted values of $T_{\mathrm{s}}$ and $T_{\mathrm{f}}$ are the default values, since these were varied in the calculations.

\begin{tabular}{ll}
\hline \hline Parameter & Reference \\
\hline$T_{\mathrm{K}}=4750 \mathrm{~K}$ & Vogt \& Hatzes (1991) \\
$T_{\mathrm{G}}=5620 \mathrm{~K}$ & Gray (1992), Cox (2000) \\
$T_{3}=4400 \mathrm{~K}$ & Gray (1992), Cox (2000); \\
& Aarum Ulvås \& Engvold (2003) \\
$C_{\mathrm{K}}=-0.31$ & Allen (1973) \\
$C_{\mathrm{G}}=-0.07$ & Allen (1973) \\
$C_{3}=-0.62$ & Allen (1973) \\
$R_{\mathrm{K}}=5.78 R_{\odot}$ & Duemmler \& Aarum (2001) \\
$R_{\mathrm{G}}=1.11 R_{\odot}$ & Duemmler \& Aarum (2001) \\
$R_{3}=0.70 R_{\odot}$ & Gray (1992), Cox (2000) \\
$d_{\mathrm{K}}=50.23$ pc & ESA (1997) \\
$d_{\mathrm{G}}=50.23$ pc & ESA (1997) \\
$d_{3}=42$ pc & Aarum Ulvås \& Engvold (2003) \\
$(B-V)_{\mathrm{K}}=0.95$ & Allen (1973) \\
$(B-V)_{\mathrm{G}}=0.70$ & Allen (1973) \\
$(B-V)_{3}=1.19$ & Fabricius \& Makarov (2000) \\
$T_{\mathrm{S}}=3455 \mathrm{~K}$ & Poe \& Eaton (1985); \\
$T_{\mathrm{f}}=5000 \mathrm{~K}$ & Vogt \& Hatzes (1991) \\
\hline
\end{tabular}

\subsection{The surface brightness method}

The relation between effective temperature $T_{\text {eff }}$, bolometric correction $C$, unreddened apparent magnitude in the $U B V$ system, $V_{0}$, and angular diameter $\phi^{\prime}$ (in milliseconds of arc) of a star,

$\log T_{\text {eff }}+0.1 C=4.2207-0.1 V_{0}-0.5 \log \phi^{\prime}$,

was derived by Barnes \& Evans (1976) using the luminosity formula $L \propto D^{2} T_{\text {eff }}^{4}$, where $D$ is the stellar linear diameter, and the Sun as calibration point.

We used Eq. (1) to derive the unreddened apparent $V$ magnitude $V_{i}$ of component $i$ in the UX Ari system. From the apparent magnitudes of each component we could derive the flux $f_{i}\left(V_{i}\right)$ of each component and the magnitude of the whole UX Ari system:

$V=-2.5 \log \left(\sum f_{i}\left(V_{i}\right)\right)$

When a dark spot with flux $f_{\mathrm{s}}$ and a bright facular area with flux $f_{\mathrm{f}}$ covered fractions $s$ and $f$, respectively, of the $\mathrm{K}$ star surface, the K star flux $f_{\mathrm{K}}\left(V_{\mathrm{K}}\right)$ was replaced by

$s f_{\mathrm{s}}\left(V_{\mathrm{s}}\right)+f f_{\mathrm{f}}\left(V_{\mathrm{f}}\right)+(1-s-f) f_{\mathrm{K}}\left(V_{\mathrm{K}}\right)$.

The UX Ari $B$ magnitude was calculated similarly, using tabulated $B-V$ values appropriate for each component's effective temperature to derive $B_{i}$ from $V_{i}$.

The calculations of the surface brightness method required several parameters of the UX Ari components as well as the spots and facular areas to be determined. Table 1 summarizes the parameters that were used.
Table 2. All available $B$ and $V$ measurements of UX Ari. The second column gives the number of measurements.

\begin{tabular}{lrl}
\hline \hline Equinox & $N$ & Source \\
\hline $1972.10-1972.99$ & 59 & Hall et al. (1975) \\
$1974.75-1975.10$ & 61 & Hall (1977) \\
$1975.93-1983.11$ & 89 & Sarma \& Prakasa Rao (1984) \\
$1984.96-1988.19$ & 46 & Mohin \& Raveendran (1989) \\
$1984.00-1987.99$ & 302 & Strassmeier et al. (1989) \\
$1989.08-1994.19$ & 73 & Raveendran \& Mohin (1995) \\
$1995.11-1995.15$ & 17 & Padmakar \& Pandey (1996) \\
$1987.87-2002.23$ & 1174 & Aarum Ulvås \& Henry (2003) \\
\hline
\end{tabular}

\subsection{The Planck function method}

Gray (1992) gives this expression for $B-V$ of a star:

$B-V=-2.5 \log \left(\frac{\int F_{\lambda} W_{B}(\lambda) \mathrm{d} \lambda}{\int F_{\lambda} W_{V}(\lambda) \mathrm{d} \lambda}\right)+0.710$,

where $F_{\lambda}$ is the flux at wavelength $\lambda$, and $W_{B}(\lambda)$ and $W_{V}(\lambda)$ are the spectral response functions for the $B$ and $V$ magnitudes, respectively. The formula in Eq. (2) is empirically corrected for non-Planckian deviations of the fluxes.

We ignored limb darkening effects in our calculations. Further, instead of the flux integrals, we used the flux at the core wavelengths of the $B$ and $V$ spectral windows, i.e. $F\left(\lambda_{B}\right)$ and $F\left(\lambda_{V}\right)$, where $\lambda_{B}=4400 \AA$ and $\lambda_{V}=5480 \AA$. The fluxes $F\left(\lambda_{B}\right)$ and $F\left(\lambda_{V}\right)$ were approximated using the Planck functions and radii of the three UX Ari components.

When a spot at temperature $T_{\mathrm{s}}$ and a facular area at temperature $T_{\mathrm{f}}$ covered portions $s$ and $f$, respectively, of the K star surface area, the K star flux $R_{\mathrm{K}}^{2} B_{\lambda_{V}}\left(T_{\mathrm{K}}\right)$ was replaced by

$s R_{\mathrm{K}}^{2} B_{\lambda_{V}}\left(T_{\mathrm{s}}\right)+f R_{\mathrm{K}}^{2} B_{\lambda_{V}}\left(T_{\mathrm{f}}\right)+(1-s-f) R_{\mathrm{K}}^{2} B_{\lambda_{V}}\left(T_{\mathrm{K}}\right)$.

Our simplifications of Eq. (2) left us with a rather simple model to work with. However, we found that this model was sufficient to reproduce the observed changes in $B-V$ and $V$ for the various sets of parameter values that were used in our calculations.

We used the temperatures and radii listed in Table 1 in the Planck function method.

\subsection{Comparison of the modelling results to observed photometry}

The observed changes in $V$ and $B-V$ are results of the rotation of the spotted K star surface. Our models consider cases where the active region contains, respectively, dark spots, and spots surrounded by a facular region of a somewhat higher temperature than the surrounding photosphere. The model mimics the rotational modulation by varying the relative area of the spots and faculae.

Table 2 summarizes all the observations that were included in our study. For comparison with the observations, we derived $\Delta V$ in the observations in the sense observed value minus 


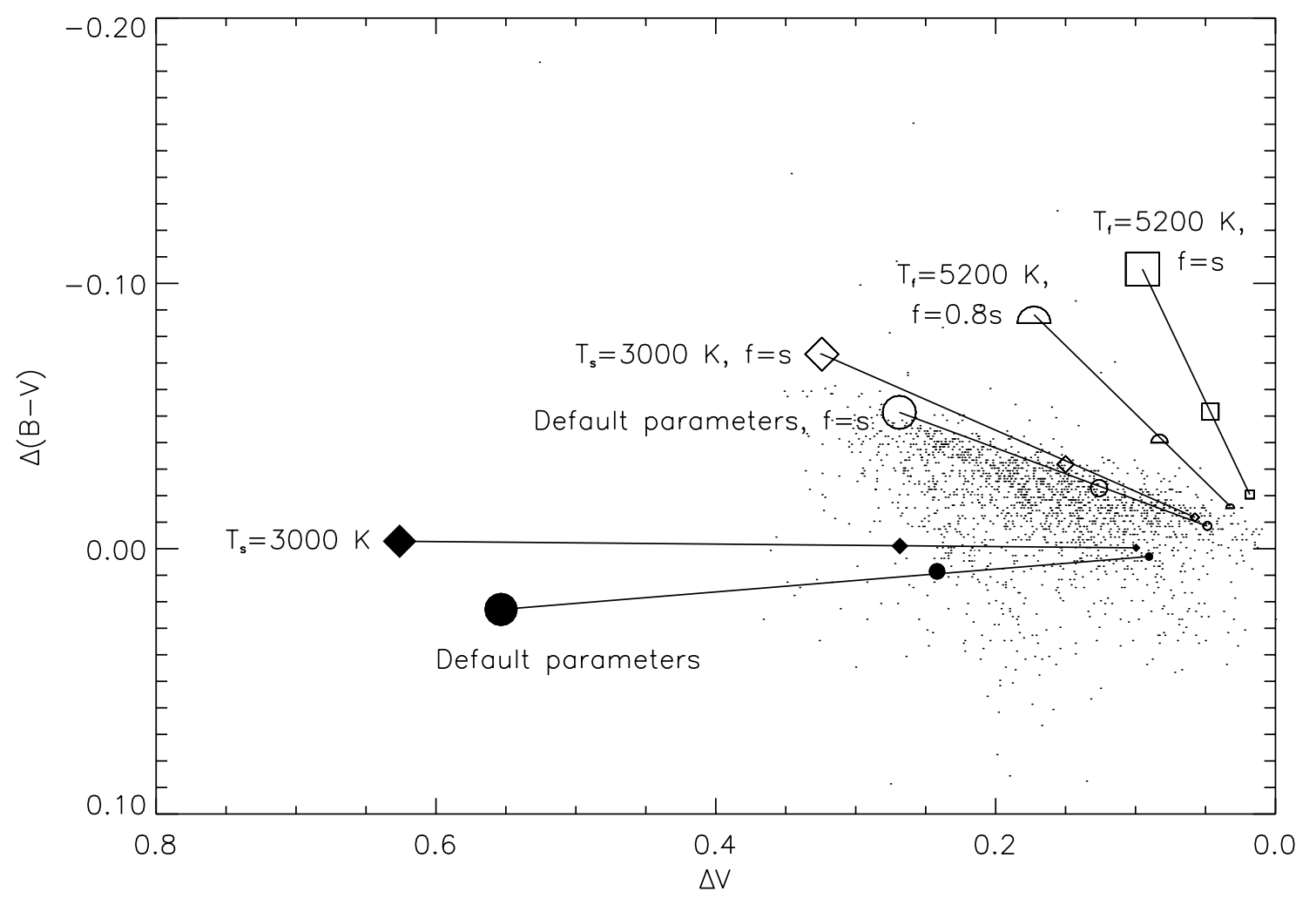

Fig. 2. Theoretical calculations, using the surface brightness method, of $\Delta V$ and $\Delta(B-V)$ of the UX Ari system relative to the UX Ari system where the $\mathrm{K}$ star is unspotted. For the observations, represented by dots, $\Delta V$ is taken relative to the brightest $V$ measurement, and $\Delta(B-V)$ is taken relative to the mean $B-V$ value of the measurements having $\Delta V<0.05$.

the brightest observed value, and $\Delta(B-V)$ in the sense observed $B-V$ minus the average $B-V$ of the observations having $\Delta V<0.05$. The brightest $V$ measurement represents UX Ari as close to unspotted as we can get from the data.

The results of our calculations using the surface brightness method and the observations are presented in Fig. 2. Observations are shown as dots. Calculations are represented by various plotting symbols, respectively open and filled, and three different sizes. All filled symbols represent calculations where only spots were included, and no facular areas. All open symbols represent calculations where both spots and facular areas were included. Small symbols represent calculations where $s=0.1$ (when no faculae were included) or $s=f=0.1$, medium-sized symbols represent $s=0.25$ or $s=f=0.25$, and large symbols represent $s=0.5$ or $s=f=0.5$. In the calculations, one of the parameters $T_{\mathrm{s}}$ or $T_{\mathrm{f}}$ was varied at a time. It is explained in the plot which symbol corresponds to which parameter. Filled symbols connected by a straight line represent calculations where only the spot coverage factor was varied. Open symbols connected by a straight line represent calculations where the spot coverage factor and the faculae coverage factor were both varied whilst maintaining the relation $s=f$. The straight lines represent gradients of the differential colour index against the differential magnitude for various sets of parameter values.

The results of the Planck function method are similar to the results of the surface brightness method, except that the facular temperature is $50 \mathrm{~K}$ higher in the former.

\section{Discussion of the results}

\subsection{Models with dark spots}

Models with spots only, and no faculae, are represented by filled symbols in Fig. 2. The calculations using the default parameters of Table 1 are represented by circles. Diamonds represent calculations with lower than default spot temperature.

The effect of dark spots is to make the flux of the $\mathrm{K}$ star fainter and redder. The reddening is only partly compensated by the bluer flux of the hotter $\mathrm{G}$ star, unless the relative flux level of the $\mathrm{K}$ star compared to the $\mathrm{G}$ star is below a certain level. We have determined the $\mathrm{K}$ star parameters for which the $\mathrm{G}$ star flux will over-compensate for the reddening by the spots, thereby bringing the calculated gradient in $\Delta(B-V)$ versus $\Delta V$ closer to the mean distribution of the observations in Fig. 2. We have found that this can be accomplished by lowering the spot temperature (see the filled symbols in Fig. 2). At the same time one needs to decrease the $\mathrm{K}$ star radius in order for the calculation results to stay within the observed range in $\Delta V$. However, a good fit to the mean distribution will require $R_{\mathrm{K}}=1 R_{\odot}$, $T_{\mathrm{s}}=1000 \mathrm{~K}$ and $s=1$, i.e. the entire visible hemisphere of the $\mathrm{K}$ star is covered by the spot. This solution implies that the active component is quite different from a $\mathrm{K}$ star, and it disagrees strongly with the flux ratio between the $\mathrm{K}$ star and the $\mathrm{G}$ star deduced from high resolution spectroscopic observations (Aarum Ulvås \& Engvold 2003). This solution consequently seems rather unlikely. 
Raveendran \& Mohin (1995) argue that the observed change in $B-V$ with fainter $V$ can be explained if the $\mathrm{K}$ star is relatively small. They claim that the magnitude difference between the two components, $V_{\mathrm{K}}-V_{\mathrm{G}}=-0.2$ at maximum light, which would imply $F_{\mathrm{K}} / F_{\mathrm{G}}=1.2$. In comparison, the default parameter values in Table 1 yield $F_{\mathrm{K}} / F_{\mathrm{G}}=11$ with both methods, which is a substantially higher flux ratio. Furthermore, we find that the Raveendran \& Mohin (1995) parameter values cited above do reproduce the observed variation in $B-V$, but since the $\mathrm{K}$ star is so small, the corresponding variation in $V$ is only $20 \%$ of the observed maximum darkening of the system.

\subsection{Models with dark spots and bright faculae}

Introducing hot facular areas in addition to cool spots on the surface of the K star (open symbols in Fig. 2) has a dramatic effect on the relation between $\Delta(B-V)$ and $V$. In all cases where faculae have been included, the gradient has the same direction as the observed one. Also, the change in magnitude decreases when facular areas are introduced, although it is still comparable to the observed range.

The UX Ari $V-R$ colour index shows the same relation with $V$ as $B-V$ does; $V-R$ increases (lower value) when $V$ decreases, as is the case with $B-V$ (Padmakar \& Pandey 1996). The relation between $V-R$ and $V$ is also reproduced by our model calculations only when introducing facular areas. This result is obtained by both methods.

A higher facular temperature (open squares in Fig. 2) increases the gradient $\Delta(B-V) / \Delta V$. Decreasing the relative area of the faculae whilst maintaining their higher temperature (open half circles in Fig. 2) has the opposite effect. There is thus an ambiguity between $T_{\mathrm{f}}$ and $f$ that our simple model cannot solve. The answer may be obtained from Doppler imaging studies of the active $\mathrm{K}$ star.

Our simplified "spot only" models fail to reproduce the observed relation between $\Delta(B-V)$ and $\Delta V$. Only by introducing hot, bright facular areas in addition to cool, dark spots on the surface of the $\mathrm{K}$ star, can the observed gradient be reproduced.

\section{Concluding remarks}

In this letter, we have presented the results from a simplified modelling of the observed variation of the $B-V$ colour index versus $V$ magnitude for the multiple UX Ari system. Our results may be summarized as follows:

- Models that include dark spots being surrounded by bright faculae on the active $\mathrm{K}$ star reproduce successfully the observed variations over a typical rotational period.

- The observed variation range of $\Delta V$ versus $\Delta(B-V)$ of UX Ari implies that the relative spot and facular areas range from 0 to 0.5 .
- Various combinations of the relative area and temperature of the stellar faculae reproduce the observed relations. Results from Doppler imaging of the UX Ari primary component may presumably remove this ambiguity.

- Models without faculae reproduce the colour variations only if the relative brightness of the active $\mathrm{K}$ star is substantially less than the hotter G star of the system. These models imply a K star with an unacceptably low flux level and must therefore be discarded.

- It is noted, however, that a few of the observed points in Fig. 2 could agree with cases without faculae and a relatively small spot area.

Acknowledgements. VAU acknowledges financial support from Institute of Theoretical Astrophysics, University of Oslo, and support from WesternGeco.

\section{References}

Aarum Ulvås, V., \& Engvold, O. 2003, A\&A, in press Aarum Ulvås, V., \& Henry, G. W. 2003, A\&A, submitted

Allen, C. W. 1973, Astrophysical quantities, 3rd edn. (University of London: Athlone Press)

Barnes, T. G., \& Evans, D. S. 1976, MNRAS, 174, 489

Carlos, R., \& Popper, D. M. 1971, PASP, 83, 504

Cox, A. N., ed. 2000, Allen's Astrophysical Quantities (New York: Springer)

Duemmler, R., \& Aarum, V. 2001, A\&A, 370, 974

ESA 1997, The Hipparcos and Tycho catalogues, Vols. 1-17, ESASP-1200

Fabricius, C., \& Makarov, V. V. 2000, A\&A, 356, 141

Gray, D. 1992, The observation and analysis of stellar photospheres, 2nd edn. (Cambridge University Press)

Hall, D. S. 1977, Acta Astron., 27, 281

Hall, D. S., Montle, R. E., \& Atkins, H. L. 1975, Acta Astron., 25, 125

Mohin, S., \& Raveendran, A. V. 1989, JApA, 10, 35

Padmakar, S. P., \& Pandey, S. K. 1996, Ap\&SS, 235, 337

Poe, C. H., \& Eaton, J. A. 1985, ApJ, 289, 644

Raveendran, A. V., \& Mohin, S. 1995, A\&A, 301, 788

Rodonò, M., \& Cutispoto, G. 1992, A\&AS, 95, 55

Sarma, M. B. K., \& Prakasa Rao, B. V. N. S. 1984, JApA, 5, 159

Strassmeier, K. G., Hall, D. S., Boyd, L. J., \& Genet, R. M. 1989, ApJS, 69, 141

Strassmeier, K. G., Hall, D. S., Fekel, F. C., \& Scheck, M. 1993, A\&AS, 100, 173

Vogt, S. S., \& Hatzes, A. P. 1991, in The Sun and Cool Stars: activity, magnetism, dynamos, ed. I. Tuominen, D. Moss, \& G. Rüdiger, IAU Coll., 130 (Springer-Verlag), 297

Wacker, S. W., Guinan, E. F., McCook, G. P., Lochner, J. C., \& Paczkowski, B. G. 1986, Inf. Bull. Var. Stars, 2920

Zeilik, M., Elston, R., Henson, G., Schmolke, P., \& Smith, P. 1982, Inf. Bull. Var. Stars, 2168 